\title{
Influência dos índices reprodutivos na produção de leite de vacas mestiças criadas no litoral Cearense
}

\author{
Robson Mateus Freitas Silveira ${ }^{*}$, Ângela Maria de Vasconcelos ${ }^{1}$, José Marcílio Araújo ${ }^{1}$, Josiel \\ Borges Ferreira ${ }^{2}$, Paulo Geovanny Alencar Oliveira ${ }^{1}$, Tiberyo Mendes Brito ${ }^{1}$
}

\begin{abstract}
RESUMO: Objetivou-se avaliar a relação entre os índices reprodutivos (intervalo entre parto e período de serviço) e a produção de leite (produção por lactação, duração da lactação e persistência de lactação) em vacas mestiças de médiabaixa e média produção. Foram utilizados dados de um controle leiteiro de 40 vacas mestiças, Holandês- Gir, de diferentes composições genéticas, todas multíparas (4 a 10 o número de lactações), no período de maio de 2015 a maio de 2016. Os animais foram divididos em dois grupos: Grupo 1 constituído de vacas de produção diária menor que 15 litros de leite, e Grupo 2 constituído de vacas com produção diária entre 15 e 20 litros de leite, sendo essa divisão fundamentada no princípio de que vacas de alta produção demoram mais a retornar ao estro e consequentemente pode influenciar na fertilidade. Os indicadores zootécnicos analisados foram: duração da lactação, média de produção diária, produção por lactação, intervalo entre parto (IEP) e período de serviço. Apenas o IEP não diferiu $(P>0,05)$ entre os grupos. A eficiência reprodutiva das vacas de média produção (15 até 20 litros/dia) não foi influenciada pela produção de leite, sendo mais viável ao produtor além de apresentar maior persistência de lactação.
\end{abstract}

Palavras-chave: curva de lactação, eficiência reprodutiva, lactação, nordeste.

\section{Influence of reproductive performance in milk production of crossbred cows created in Ceará.}

\begin{abstract}
The objective of this study was to evaluate the relationship between reproductive indices (interval between delivery and service period) and milk production (lactation yield, lactation duration and persistence of lactation) in medium-low and medium-production crossbreeding cows. Data from a dairy control of 40 crossbred Holstein-Gir cows of different genetic compositions, all multiparous (4 to 10 lactation), were used from May 2015 to May 2016. The animals were divided in two groups: Group 1 consisting of cows of daily production less than 15 liters of milk, and Group 2 consisting of cows with daily production between 15 and 20 liters of milk, this division being based on the principle that high production cows take longer to return to estrus and consequently may influence fertility. The zootechnical indicators analyzed were: duration of lactation, average daily production, lactation production, interval between parturition (IEP) and period of service. Only the IEP did not differ $(\mathrm{P}>0.05)$ between the groups. The reproductive efficiency of the medium-production cows (15 to 20 liters / day) was not influenced by milk production, being more viable to the producer besides presenting a higher persistence of lactation
\end{abstract}

Keywords: lactation curve, reproductive efficiency, lactation, northeast

\section{INTRODUÇÃO}

O Brasil é o quinto maior produtor mundial de leite, com uma estimativa de produção de 34,5 bilhões de litros no ano de 2015, o que equivale a uma produtividade por lactação de 1.620 litros de leite por vaca. Em comparação aos maiores produtores mundiais, com exceção da Índia, a produtividade do rebanho leiteiro nacional é considerada baixa, uma vez que a média dos Estados Unidos, maior produtor de leite do mundo, é de 10.322 litros vaca $^{-1}$ lactação $^{-1}$ (USDA, 2014). Essa disparidade na produtividade da pecuária leiteira nacional está relacionada com diversos fatores, como: a ineficiência reprodutiva, representada pela idade avançada ao primeiro parto e pelo longo intervalo entre partos; o baixo potencial genético dos animais, resultando em baixa produção lactacional com lactações curtas e de baixa persistência na produção; instalações que não proporcionam o conforto térmico aos animais e a falta de um manejo sanitário efetivo. (Ferreira, 1991; Facó et al., 2002; Leal et al., 2013; Borges et al., 2015).

A eficiência reprodutiva é o fator que, isoladamente, mais afeta a produtividade e a lucratividade de um rebanho, entretanto há muitas barreiras para melhorar, pois ocorrem perdas reprodutivas desde a concepção até o parto. Com a redução da produção de leite haverá um maior intervalo entre as lactações, o que causaria um maior período seco, ou seja, um maior número de vacas secas em relação a vacas em produção. (Bergamaschi et al., 2010)

A intensa seleção nas características produtivas nas últimas décadas foi responsável pelo declínio na fertilidade de vacas leiteiras. (Kung et al, 2006).

Recebido em 16/10/2017; Aceito para publicação em 06/06/2018

${ }^{1}$ Universidade Estadual Vale do Acaraú

2 Universidade Federal Rural do Semi-Árido

*E-mail: robsonmateus1994@hotmail.com 
Esse fato pode ser explicado pela baixa herdabilidade estimada para fertilidade $(<5 \%)$ enquanto a herdabilidade das características produtivas é considerada moderada a alta (> 50\%) (Pereira et al., 2008).

Diante dos fatos expostos, o presente trabalho tem como objetivo avaliar a relação entre os índices reprodutivos (intervalo entre parto e período de serviço) e a produção de leite (produção por lactação e persistência de lactação) em vacas mestiças, Holandês- Gir, de média- baixa e média produção.

\section{MATERIAL E MÉTODOS}

Foram utilizados dados produtivos e reprodutivos de animais pertencentes a uma propriedade comercial leiteira do município de Aquiraz, no litoral leste do Ceará, zona fisiográfica de Tabuleiros Pré-Litorâneos e Várzea do Rio Pacotí, inserida na localidade de Lagoa das Canas, cuja sede se encontra a 14,23 m de altitude e nas seguintes coordenadas $3^{\circ} 54^{\prime}$ 05" Sul e $38^{\circ} 23^{\prime} 28^{\prime}$ ' Oeste. O clima da região, de acordo com a classificação de Koppen - Geiger, é do tipo Aw, (tropical quente com estação semiúmida).

A pluviometria é marcada pela irregularidade das chuvas, cuja distribuição se dividem em dois períodos, um seco e outro chuvoso, apresentando valores de precipitação anual entre 1.000 e 1.380 $\mathrm{mm}$, temperatura média de $26^{\circ} \mathrm{C}$ (Silva e Colares, 2012), umidade relativa média do ar de $78 \%$, com tempo de insolação de 269,4 dias/ano e velocidade média anual dos ventos de $3,6 \mathrm{~km} / \mathrm{h}$ (IPECE, 2010)
Os dados produtivos e reprodutivos analisados foram extraídos do período compreendido de maio de 2015 a maio de 2016, a partir da escrituração zootécnica de 40 vacas mestiças, Holandês- Gir, de diferentes grupos genéticos, com produção média diária variando de 10 a 20 litros, todas multíparas (4 a 10 lactações).

Baseando-se nesses dados, os animais foram divididos em dois grupos: Grupo 1 - vacas de produção diária menor que 15 litros de leite; Grupo 2 - vacas com produção diária entre 15 e 20 litros de leite, na pressuposição de que vacas de alta produção demoram mais a retornar ao estro, o que pode influenciar na fertilidade (Simões, 2010). A produção média diária foi obtida dividindo-se a produção total de leite da lactação pelo número de dias da lactação

O sistema de criação era o semi-intensivo com fornecimento de $350 \mathrm{~g}$ de concentrado comercial (Tabela 1) para cada litro leite produzido no dia anterior, dividido em duas porções iguais, uma fornecida na primeira ordenha diária (03:00h) e outra na segunda ordenha do dia (15:00). Após a primeira ordenha, os animais permaneciam em pastejo rotacionado, constituído pelo consórcio dos capins Cost Cross (Cynodon dactylon), Mombaça (Panicum maximum cv. Mombaça) e Tanzânia (Panixum maximum cv. Tanzânia), até a segunda ordenha. Após esta ordenha, os animais eram estabulados em currais próximos à central de manejo, para o fornecimento, no cocho, de cana-deaçúcar. (Saccharum officinarum) e capim Cost Cross e Napier (Pennisetum purpureum) picados fresco no cocho.

Tabela 1 - Composição nutricional da ração oferecida para as vacas leiteiras

\begin{tabular}{rcrc}
\hline Nutriente & Teor & Nutriente & Teor \\
\hline Matéria Seca (\%) & 88,91 & Zinco $(\mathrm{ppm})$ & 127,51 \\
Proteína Bruta (\%) & 18,86 & Selênio $(\mathrm{ppm})$ & 0,65 \\
Extrato Etéreo (\%) & 3,3 & Cobalto $(\mathrm{ppm})$ & 0,61 \\
Ureia (\%) & 4,7 & Iodo $(\mathrm{ppm})$ & 1,34 \\
Matéria Mineral (\%) & 5,2 & Cromo $(\mathrm{ppm})$ & 0,00005 \\
FDA (\%) & 3,53 & Cobre $(\mathrm{ppm})$ & 32,76 \\
Lignina (\%) & 0,89 & Manganês $(\mathrm{ppm})$ & 69,77 \\
Cálcio (\%) & 0,81 & Cloro $(\mathrm{ppm})$ & 0,09 \\
Fósforo (\%) & 0,55 & Ferro $(\mathrm{ppm})$ & 90,44 \\
Magnésio $(\mathrm{ppm})$ & 0,27 & Vitamina A (IU/ $\mathrm{kg})$ & 6,72 \\
Potássio $(\mathrm{ppm})$ & 0,85 & Vitamina D (IU/ $\mathrm{kg})$ & 2,65 \\
Enxofre $(\mathrm{ppm})$ & 0,29 & Vitamina E $(\mathrm{IU} / \mathrm{kg})$ & 50,44 \\
Sódio $(\mathrm{ppm})$ & 0,24 & Biotina $(\%)$ & 2,0744 \\
\hline
\end{tabular}

As vacas eram identificadas através do número do brinco e separadas em piquetes próximos às instalações ao apresentarem os primeiros sinais de cio. Após 12 horas, eram inseminadas, anotando todas as informações necessárias, retornando posteriormente ao grupo de ordenha. O diagnóstico da prenhez era realizado aos 45 a 60 dias após a inseminação.

A ordenha era realizada em uma ordenhadeira mecânica com circuito fechado, do tipo linha baixa 
duplo com 16 conjuntos. O controle leiteiro foi realizado semanalmente e a produção de leite era medida através de medidores individuais acoplados na ordenhadeira.

Os dados reprodutivos e de produção diária de leite eram anotados em fichas individuais, digitalizados e processados em software de escrituração zootécnica, resultando na estimativa dos índices reprodutivos (intervalo entre partos e período de serviço) e produtivos (média de produção, produção por lactação, duração de Lactação, persistência da lactação).

Cada índice foi calculado individualmente, separados em 2 grupos conforme produção média diária e mensurada a média e desvio padrão para cada tratamento.

Os índices calculados individualmente foram:

1. Intervalo entre partos, meses (IEP $\left.=\mathrm{P}_{\mathrm{y}}-\mathrm{P}_{\mathrm{x}}\right)$

$\mathrm{P}_{\mathrm{x}}=$ Data do parto

$\mathrm{P}_{\mathrm{y}}=$ Data do parto subsequente

2. Período de serviço, dias $\left(\mathrm{PS}=\mathrm{DG}-\mathrm{P}_{\mathrm{x}}\right)$

$\mathrm{P}_{\mathrm{x}}=$ Data do parto anterior

$\mathrm{DG}=$ Data do diagnóstico de gestação

3. $\quad$ Média de produção de leite $(\mathrm{MP}=\mathrm{PT} / \mathrm{PL})$

$\mathrm{PT}=$ Produção de leite total

PL $=$ Período lactacional
4. Produção por lactação (PPL $=$ PPL / N

$\mathrm{PPL}=$ Produção de leite da lactação

$\mathrm{N}$ = Número de animais em lactação

5. Duração de Lactação, dias $\left(\mathrm{DL}=\mathrm{S}-\mathrm{P}_{\mathrm{x}}\right)$

$\mathrm{P}_{\mathrm{x}}=$ Data do parto

$\mathrm{S}=$ Data da secagem

6. Persistência da Lactação, \% (PL = [1((volume do controle anterior - volume do controle atual) x (30dias de intervalo entre as pesagens) / volume do controle anterior] $\mathrm{x}$ 100)

$\mathrm{O}$ delineamento adotado foi $\mathrm{o}$ inteiramente casualizado (DIC), utilizando 40 lactações de 40 vacas, dividida em dois grupos conforme produção média diária com 20 lactações em cada tratamento. Os dados foram submetidos a análise de variância, utilizando o PROC GLM do SAS® $\quad$ (SAS INSTITUTE, versão 9.1). As médias entre os grupos foram comparadas considerando o nível de significância de 5\% utilizando o teste de F

\section{RESULTADOS E DISCUSSÃO}

Os valores médios dos índices produtivos e reprodutivos nos dois grupos diferiram entre si $(\mathrm{P}<0,05)$, exceto o IEP $(\mathrm{P}>0,05)$ (Tabela 3).

Tabela 3 - Índices produtivos e reprodutivos dos diferentes grupos de produção de leite

\begin{tabular}{ccc}
\hline Parâmetros & GRUPO 1 (média \pm DP) & GRUPO 2 (média \pm DP) \\
\hline Intervalo entre parto (mês) & $14,48 \pm 4,86^{\mathrm{a}}$ & $13,30 \pm 2,75^{\mathrm{a}}$ \\
Período de serviço (dia) & $152,95 \pm 123,97^{\mathrm{b}}$ & $126,80 \pm 81^{\mathrm{a}}$ \\
Média de produção (litro/vaca/dia) & $11,70 \pm 2,42^{\mathrm{b}}$ & $16,88 \pm 2,13^{\mathrm{a}}$ \\
Produção por lactação (litros) & $3141,95 \pm 962,84^{\mathrm{b}}$ & $4731,65 \pm 911,54^{\mathrm{a}}$ \\
Duração da lactação (dias) & $260,70 \pm 42,86^{\mathrm{a}}$ & $279,95 \pm 50,51^{\mathrm{b}}$ \\
\hline
\end{tabular}

Grupo 1 = Animais produzindo até 14 litros leite/dia; Grupo 2= Animais produzindo mais de 14 litros leite/dia. Médias seguidas por letras distintas na mesma linhas diferem entre si pelo teste de $\mathrm{F}$ a $5 \%$ de probabilidade

O IEP dos dois grupos apresentaram valores acima dos considerados ideais (12 meses). Isso possivelmente está relacionado às condições corporais inferiores das matrizes no momento do parto e ao déficit nutricional nas semanas subsequentes, sendo essas as principais causas de anestro pós-parto reportados na literatura. (Rangel et al., 2009). O Grupo 2 apresentou uma maior eficiência reprodutiva quando comparado com o Grupo 1, apesar de uma maior produção leiteira, duas variáveis teoricamente inversamente relacionadas (Madalena, 2007). Essa aparente contradição pode ser resultado do background genético, uma vez que os animais foram submetidos aos mesmos manejos e ambientes.

A média do IEP do Grupo 2 está nos limites estabelecidos por Bergamaschi, Machado e Barbosa
(2010) e Ferreira (2007), de 12 a 14 meses para vacas mestiças, enquanto a do Grupo 1 apresentou valores levemente superiores aos ideias estabelecidos. Os resultados encontrados neste estudo foram diferentes de Neves et al. (2011) que analisando os índices zootécnicos de 28 propriedades comerciais de leite no Perímetro Irrigado Senador Nilo Coelho, em Petrolina - PE, obtiveram valores médios do IEP de 19, 2 para vacas com diferentes padrões raciais e próximos ao de Rangel et al. (2009) trabalhando com animais de um rebanho Guzerá, no Agreste Paraibano de 1988 a 1998, observaram médias de IEP de $14,72 \pm 2,43$ meses

Níveis de produção de leite semelhantes ao deste trabalho foram encontrados em alguns trabalhos no Nordeste para vacas mestiças de diferentes grupos 
genéticos (GOMES et al., 2015; BEZERRA et al., 2010; FREITAS et al., 2014)

A DL influenciou na produção total de leite de ambos os grupos, verificando-se que quanto maior a duração da lactação maior a produção total de leite dos animais. Esses resultados também foram reportados por Neiva (1991) e Barbosa et al., (1994), que indicaram que a duração do período de lactação está diretamente relacionada à quantidade total de leite. A DL, foi maior no Grupo 2 com média de 279,95 dias. Possivelmente isso ocorreu devido à maior produção de leite na lactação e a maior participação de grau de sangue Holandês na mestiçagem desses animais. O período de lactação ideal é de 305 dias para vacas Holandesas e Jersey puras, entretanto para mestiças destas raças a DL tende a ser menor, com duração média de 270 a 280 dias (FERREIRA, 2011).

Resultados similares foram encontrados por Bezerra et al. (2010), que reportaram duração da lactação de $286,5 \pm 52,97$ dias e produção de $4.132,61 \pm 1.163,22 \mathrm{~kg}$ por lactação em vacas mestiças criadas em sistema semiintensivo no Norte do Piauí. Freitas et al. (2014) analisaram 2670 lactações de vacas Girolando no Nordeste do Brasil e verificaram uma duração de lactação de $264 \pm 98$ dias com produção total por lactação de $4094 \pm 2010$ litros de leite. Valores superiores foram reportados por MacManus et al. (2008) que observaram a produção de $2.866 \mathrm{~kg}$ em 284 dias de lactação em animais mestiços das raças Holandesa e Gir, com diferentes composições genéticas, na região do Planalto Central brasileiro. Essa superioridade possivelmente se deve ao maior grau de sangue holandês nesses animais e ao clima da região que proporcionou um maior conforto térmico aos animais

Verificou-se que os níveis de produção influenciaram no pico de lactação (Gráfico 1). O Grupo 1 apresentou o pico de lactação na quinta semana, enquanto o Grupo 2, na sétima semana. Essa variação de tempo é relatada por Vasconcelos e Santos (2007) e Cabuci et al. (2003), em que vacas de alta produção apresentaram um pico de lactação mais tardio em relação a vacas de baixa produção. Esses autores explicam que o pico tardio é um resultado do estresse fisiológico causado pelo Balanço Energético Negativo (BEN) nas vacas de maior produção. Nestes animais, o consumo de energia é inferior às exigências energéticas envolvidas na crescente produção de leite após o parto, e causa esta

Santos (2011) avaliando a curva de lactação de vacas mestiças Holandês- Zebu em Felixlândia, Minas Gerais, entre os meses de janeiro e maio de 2008, observou que estas alcançaram o pico de lactação de 19,80 litros de leite aos 34 dias, valores muitos Em relação à persistência da lactação, ambos os grupos apresentaram resultados satisfatórios (Gráfico 2), visto que Freitas (2005) classifica uma vaca de boa persistência, quando apresenta valores aproximados à $90 \%$, ideal para vacas de raças especializadas. Entretanto, para vacas mestiças essa taxa pode cair para $80 \%$ ou até menos. Assim, verificou-se que as matrizes do Grupo 1 apresentaram um valor superior ao estabelecido para suas características raciais, enquanto que as matrizes do Grupo 2 demostraram um percentual superior ao padrão e próximo ao ideal para vacas com aptidão leiteira.

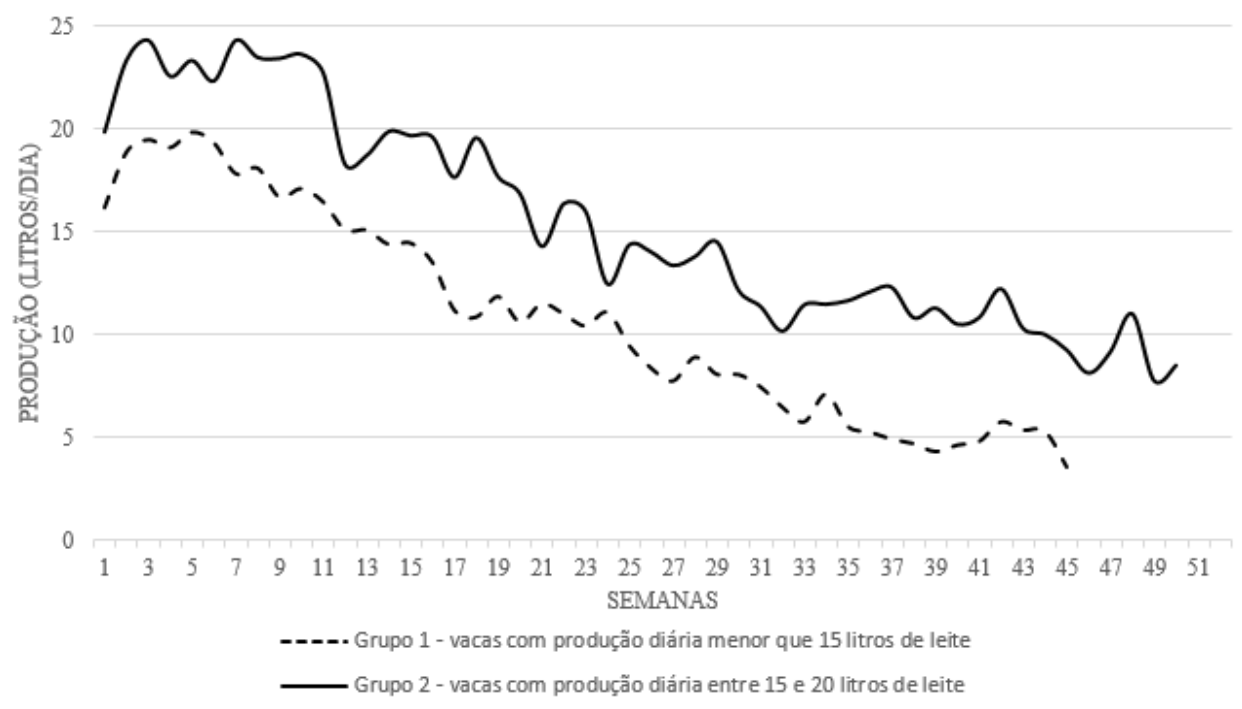

Gráfico 1 - Curvas de lactação das vacas mestiças Holandês- Gir dos diferentes grupos de produção de leite 


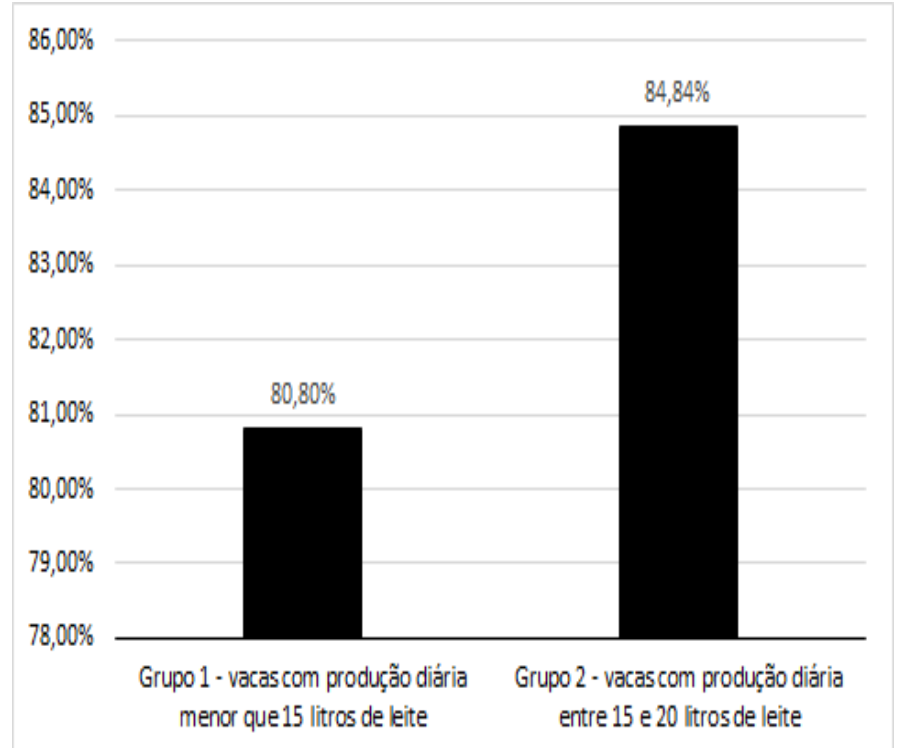

Gráfico 2- Persistência da lactação dos diferentes grupos de produção de leite

\section{CONCLUSÃO}

1 - A eficiência reprodutiva das vacas de média produção (15 até 20 litros/dia) não foi influenciada pela produção de leite.

2- Vacas com média produtiva de até 15 litros de leite são mais viáveis ao sistema de produção, podem mantém uma boa relação entre os índices produtivos e reprodutivos.

\section{AGRADECIMENTOS}

À Empresa M \& E Consultoria e Agropecuária LTDA que em parceria Paulo Geovanny Alencar Oliveira disponibilizou os dados de uma propriedade leiteira para a realização desse trabalho

\section{REFERÊNCIAS}

BARBOSA, S. P. B; MANSO, H. C; SILVA, L. O.C, Estudo do período de lactação de vacas holandesas no estado de Pernambuco, Revista Brasileira de Zootecnia, Viçosa-MG, v. 23, n.3, v. 23, p. 465- 475, 1994.

BERGAMASCHI, M. C. M. A; MACHADO, R.; BARBOSA, R. T. Eficiência reprodutiva das vacas leiteiras. Juiz de Fora, MG: Embrapa Gado de leite, Circular Técnica, n. 64, 2010.

BEZERRA, E. E. A.; MAGALHÃES, J. A.; AZEVÊDO, D. M. M. R.; PEREIRA, R. G. de A.; TOWNSEND, C. R.; COSTA, N. de L. Produção de leite e intervalo entre partos de um rebanho de vacas mestiças no Norte do Piauí. PUBVET, Londrina- PR, v. 5, n. 1, ed. 148, Art. 992, 2011.
COBUCCI, J.A.; EUCLYDES, R. F.; PEREIRA, C.S. Persistência na lactação - uma revisão. Archivos Latinoamericanos de Produccion Animal, v.11, n.3, p.163-173, 2003.

FERREIRA A. M. Manejo reprodutivo e sua importância na eficiência da atividade leiteira. Coronel Pacheco: Embrapa-CNPGL, 1991. 47p. (Embrapa/CNPGL, 46)

FERREIRA, A. M.; MIRANDA, J. E. C. Medidas de eficiência da atividade leiteira: índices zootécnicos para rebanhos leiteiros. $1^{\text {a }}$ Ed. Juiz de Fora, MG: Embrapa Gado de leite, v.1, n.54, p. 8, 2007.

FERREIRA, F. C. Controle Leiteiro: lucro para o produtor. Porto Velho- RO: Embrapa Rondônia. 2011. Disponível em: <http://ainfo.cnptia.embrapa.br/digital/bitstream/item/427 01/1/folder-leite.pdf $>$. Acesso em: 19 de maio de 2016.

FREITAS, A. F. Persistência da lactação: uma referência absoluta. Publicações Rehagro: Artigos técnicos. 2005. Disponível em: <http://www.rehagro.com.br/siterehagro/publicacao.do?c dnoticia=1001>. Acesso em: 30 de junho de 2016.

FREITAS, A. F. de; MARTINS, M. F; ARBEX, W.; SANTOS, K. C. L. dos; SILVA, M. G. B. da. Desempenho do Girolando nos rebanhos do Nordeste do Brasil. Revista O Girolando, v. 16, n. 94, p. 24-25, 2014.

GOMES, N. J. G.; SARAIVA, C. A. S.; NETO, S. G.; ALCOFORADO, A. De A. T.; SILVA, E. P. da; ALVES, A. dos S.; GOMES, T. B. B.; FIGUEIREDO, P. R. N. de. Avaliação do Desempenho Zootécnico em uma Unidade de Produção de Leite no Brejo Paraibano. In: X 
Congresso Nordestino de Produção Animal, 2015, Teresina. Anais... X CNPA, 2015.

INSTITO DE PESQUISA E ESTRATÉGIAS ECONÔMICAS DO CEARÁ (IPECE). Perfil Básico do Município de Aquiraz. 2010. Disponível em: <http://www.ipece.ce.gov.br/publicacoes/perfil_basico/P BM_2006/Aquiraz.pdf>. Aceso em: 10 de junho de 2016

MADAlenA, F. E. Problemas dos rebanhos leiteiros com genética de alta produção - Revisão bibliográfica. Belo Horizonte: Universidade Federal de Minas Gerais, 2007. 33 p.

MARQUES JR, A. de P. Manejo Reprodutivo de Bovinos. In: VI Congresso Norte Nordeste de Reprodução Animal, 2012, Fortaleza. Anais... VI CONERA, 2012.

MCMANUS, C; TEIXEIRA, R. de A. T.; DIAS, L. T.; LOUVANDINI, H.; OLIVEIRA, ELIANDRA M. B., Características produtivas e reprodutivas de vacas Holandesas e mestiças Holandês x Gir no Planalto Central. Revista Brasileira de Zootecnia, Viçosa-MG, v.37, n.5, p.819-823, 2008.

NEIVA, R.S. Bovinocultura de Leite. Lavras - MG, ESAL/FAEPE, 1991. 238p

NEVES, A. L. A.; PEREIRA, L. G. R.; SANTOS, R. D. dos; ARAÚJO, G. G. L. de; CARNEIRO, A. V.; MORAES, S. A.; SPANIOL, C. M. O.; ARAGÃO, A. S. L. de. Caracterização dos produtores e dos sistemas de produção de leite no perímetro irrigado de Petrolina/PE.
Revista Brasileira de Saúde Produção Animal, Salvador- BA, v.12, n.1, p.209-223, jan/mar, 2011

PEIXOTO JUNIOR, K.C., FIRMIANO, A.C. e CRESPILHO, A.M. Relação entre reprodução e produção de bovinos de leite. PUBVET, Londrina- PR, v. 7, n. 24 , 2013

RANGEL, A. H. do N.; GUEDES, P. L. C.; ALBUQUERQUE, R. P. F.; NOVAIS, L. P.; LIMA JÚNIOR, D. M. de. Intervalo entre parto e período de serviço de vacas Guzerá. Revista Verde de Agroecologia e Desenvolvimento Sustentável, Mossoró- RN, v.4, n.3, p. 21- 25, 2009.

SANTOS, S.A. Curvas de lactação e consumo de vacas F1 Holandês $x$ Zebu em pastejo e em confinamento. 2011. p. 213. Tese (Doutorado em Zootecnia). Universidade Federal de Viçosa, Viçosa- MG.

SIMÕES, A. R. Eficiência reprodutiva em vacas mestiças leiteiras em municípios do nordeste paraenses. 2010. p. 63. Dissertação (Mestrado em Ciências Agrárias e Desenvolvimento Rural). Universidade Federal do Pará, Belém- PA..

SILVA, J. G. da; COLARES, J. Q. dos. Hidrogeologia da faixa costeira de Aquiraz- CE. In: 1st Joint World Congress on Groundwater, 2000, Fortaleza. Anais...1st Joint World Congress on Groundwater. 2000. 